(Aus dem physiologischen Institut der Universität Zürich.)

\title{
Beiträge zur physikalischen Chemie der Erregung und der Narkose.
}

Von

\section{Rudolr Höber.}

(Mit 14 Textfiguren.)

Mehrere Versuchsreihen haben mich zu der Ansicht geführt, dass beim Erregungsvorgang die Kolloide, welche jede erregbare Substanz wie jeder Protoplast überhaupt enthält, eine Rolle spielen, indem sich während der Erregung Änderungen in der Konsistenz oder im Lösungszustand dieser Kolloide vollziehen. Dieser Kolloidprozess kann nach meiner Meinung ebenso als Charakteristikum des Erregungsvorgangs gelten, wie der als Aktionsstrom bezeichnete elektrische Prozess; denn es lässt sich zeigen, dass, wenn der Kolloidprozess zum Wegfall gebracht wird, auch die Erregung nicht mehr zustande kommt. Die Gründe für meine Ansicht sind im Folgenden enthalten.

\section{Kuhestrom und Kolloidzustand beim Muskel.}

Ich habe früher ${ }^{1}$ ) gezeigt, dass, wenn man unversehrte Sartorien vom Frosch lokal mit den isotonischen Lösungen der neutralen Alkalisalze behandelt, und von behandelter und einer nicht behandelten Stelle mit unpolarisierbaren Ringer-Calomel-Elektroden ableitet, Ruheströme nachweisbar sind, deren elektromotorische Kraft nach Grösse und Richtung mit der Art des Alkalisalzes wechselt. Die Resultate lassen sich folgendermaassen tabellieren:

1) Pflüger's Arch. Bd. 106 S. 599.1905. 


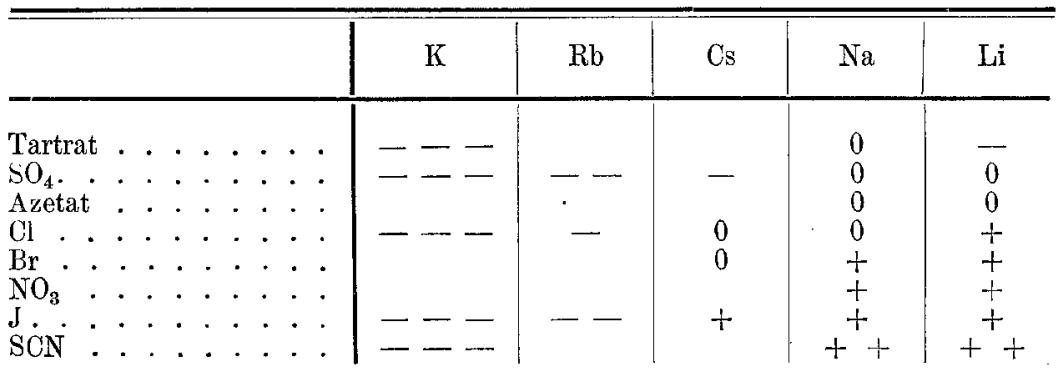

In der Tabelle bedeutet ein Minuszeichen, dass die mit Salz behandelte Stelle "negativ" wird, d. h. dass ein regulärer Ruhestrom auftritt, der im äusseren Schliessungsbogen von der nicht behandelten zur alterierten Stelle fliesst. Ein Pluszeichen deutet dann das Auftreten eines entgegengesetzten, von der alterierten zur normalen Stelle fliessenden Stromes an, und das Nullzeichen soll besagen, dass das betreffende Salz gar keine stromentwickelnde Fähigkeit besitzt. Die Zahl der Zeichen gibt einen ungefähren Maassstab für die Grösse der auftretenden E.-M.-K. ab.

Man ersieht nun aus der Tabelle, dass die Fähigkeit der Kationen, einen regulären Ruhestrom $z \mathfrak{u}$ erzeugen, offenbar ansteigt in der Reihenfolge: $\mathrm{Li}<\mathrm{Na}<\mathrm{Cs}<\mathrm{Rb}<\mathrm{K}$, während die entsprechende Fähigkeit der Anionen steigt in der Reihenfolge: $\mathrm{SCN}<\mathrm{I}<\mathrm{NO}_{3}$, $\mathrm{Br}<\mathrm{Cl}<$ Azetat $<\mathrm{SO}_{4}<$ Tartrat.

Fragt man nach einer Erklärung dieser Salzwirkungen, so lässt sich geltend machen, dass die Stufenfolge der Anionen ganz und gar übereinstimmt mit der Abstufung, welche die Anionen hinsichtlich ihrer Fähigkeit, hydrophile Kolloide auszusalzen oder deren Quellungszustand zu beeinflussen, zeigen, und dass wenigstens auch die Kationenreihe $\mathrm{Li}<\mathrm{Na}<\mathrm{K}$ als Wirkungsreihe bei den Kolloiden bekannt ist, während Angaben über den Einfluss von $\mathrm{Rb}$ und $\mathrm{Cs}$ auf die Kolloide bisher fehlen. Ich habe deshalb die Hypotbese aufgestellt, dass Richtung und Grösse der E.-M.-K. der unter der Einwirkung der Salze auftretenden Ruheströme mit einer je nach der Art des Salzes verschiedenen Einwirkung auf die Kolloide der Muskelprotoplasten zusammenhängen; aus Gründen, auf welche ich hier nicht noch einmal zurückkommen will, hat man dabei in erster Linie an die die Plasmahaut formierenden Kolloide zu denken ${ }^{\mathbf{1}}$ ).

1) Siehe hierzu auch: Höber, Physik. Chemie der Zelle und der Gewebe, 2. Aufl., S. $260 \mathrm{ff}$, und 273.1906. 
Das Entstehen von E.-M.-K. durch die Salzwirkung kann man dann im Anschluss an Bernstèin's Membrantheorie des Ruhestroms auf Änderungen der Ionenpermeabilität der Plasmahaut durch die Kolloidzustandsänderungen in ihr zurückführen.

Diese Hypothese habe ich jetzt zunächst durch einige ergänzende Untersuchungen an Kolloiden zu stützen versucht. Sie erstreckten sich erstens auf die relative Fällungskraft von Rubidium- und Caesiumsalzen, zweitens auf die Aussalzbarkeit des Lezithins. Denn wenn man die physiologische Kationenreihe: $\mathrm{Li}<\mathrm{Na}<\mathrm{Cs}<\mathrm{Rb}<\mathrm{K}$ ansieht, so ist daran die Stellung des Cs so auffallend und abweichend von seiner Stellung seiner chemischen Natur nach, dass die Ermittelung des Platzes, welchen das Cs in der Reihe der Kolloidfällungsmittel einnimmt, wohl einen Prüfstein für die ausgesprochene Hypothese eines Zusammenhangs zwischen Ruhestrom und Kolloidkonsistenz abgeben kann. Die Untersuchung des Lezithins ist aber deshalb von besonderem Interesse, weil nach allem, was wir darüber wissen, das Lezithin wohl eine Hauptkomponente der Plasmahaut ausmacht ${ }^{1}$ ).

Beim Lezithin habe ich nun folgendes gefunden: 1. das Anionenfällungsvermögen nimmt zu in der Reihenfolge:

$$
\mathrm{I}<\mathrm{SCN}<\mathrm{Br}, \mathrm{NO}_{3}<\mathrm{Cl}<\text { Azetat }<\mathrm{SO}_{4},
$$

also in derselben Reihenfolge wie bei anderen hydrophilen Kolloiden und in derselben Reihe, in der die Anionen die physiologische Erscheinung des Rubestromes abstufen;

2. das Kationen-Fällungsvermögen nimmt im allgemeinen zu in der Reihenfolge:

$$
\mathrm{K}<\mathrm{Rb}<\mathrm{Li}<\mathrm{Cs}, \mathrm{Na} .
$$

Diese Reihe entspricht ziemlich genau der physiologischen Kationenreihe, und vor allem zeigt sich auch, dass $\mathrm{Cs}$ nicht mit $\mathrm{K}$ und $\mathrm{Rb}$ zusammen rangiert, sondern der Gruppe $\mathrm{Li}, \mathrm{Na}$ angehört. $\mathrm{Da}$ diese Art der Anordnung bei anderen Neutralsalzwirkungen als bei der Kolloidaussalzung bisher nicht bekannt ist, so erblicke ich darin eine starke Stütze der Hypothese vom Zusammenhang zwischen Ruhestrom und Kolloidzustand.

1) Von den Ergebnissen meiner Kolloiduntersuchungen teile ich im folgenden nur dasjenige mit, was direkt auf die physiologischen Fragen Bezug hat. Im äbrigen verweise ich auf die eingehendere Abhandlung in Hofm eis ter's Beiträgen. 
Ergänzende Untersucbungen über die Fällbarkeit von $\mathrm{H} u ̈ h \mathrm{~h}$ er e i weiss lieferten bei der Fällung mit Chloriden die Kationenreihe;

$$
\mathrm{Li}<\mathrm{Na}, \mathrm{Cs}<\mathrm{Rb}<\mathrm{K},
$$

also wiederum die physiologische Reihe! ${ }^{1}$ ) Beim Eiweiss liegen die Verhältnisse allerdings für die Ausdeutung der Versuche zugunsten meiner Hypothese insofern schwieriger als beim Lezithin, als bei Fällung des Eiweisses mit anderen Salzen als den Chloriden auch andere Kationenreihen auftreten. Wie das theoretisch zu erklären ist, darauf will ich hier nicht eingehen ${ }^{2}$ ). Wesentlich erscheint mir hier jedoch, dass die physiologische Kationenreihe gerade bei der Fällung mit Chloriden auftritt; denn auch bei meinen Versuchen an den frischen Muskeln überwogen auf alle Fälle, mit welchem Salz auch immer ein Muskel zur Stromerzeugung lokal behandelt wurde, in den Interfibrillärräumen die normaler Weise dort schon vorhandenen Chlorionen, da sie während der kurzen Zeit der.Einwirkung der stromerzeugenden Salzlösung nicht Zeit finden, aus dem Muskel herauszudiffundieren. Es unterstützen als o a u ch die Ei weissversuche die Ruhestrom-Hypothese.

\section{Ruhestrom und Erregbarkeit beim Maskel.}

Meinen Ruhestrom-Messungen kommt, wie ich glaube, eine ziemlich erhebliche Bedeutung für die Theorie des Erregungsvorganges zu; deshalb ist es notwendig, zunächt einen naheliegenden Einwand gegen die Messungen zu diskutieren, welcher jüngst von v. Frey ${ }^{3}$ ) mit folgenden Worten erhoben worden ist: "Eine Diskussion der Versuche Höbers ist dadurch erschwert, dass der den Muskel ableitende Bogen von vorn herein unsymmetrisch ist und daher in sich schon Potentialdifferenzen enthält, die, wie die Versuche von OkerBlom zeigen, nicht vernachlässigt werden dürfen."

Es ist in der Tat vollkommen richtig - und ich selbst bin mir

1) Auffallen wird, dass die Kationenreihe beim Eiweiss gerade umgekehrt lautet, wie beim Lezithin. Es erinnert diese Erscheinung an die Umdrehung der Anionenreihe beim Eiweiss durch Zusatz kleiner Mengen Säure oder Lange, wie es von Posternak und Pauli beobachtet wurde. Durch dieselben Mittel lässt sich, wie ich in der eingehenderen Abhandlung in $\mathrm{H}$ ofmeister's Beiträgen zeige, auch die Kationenreihe leicht umdrehen.

2) Siehe darüber die eingehendere Abhandiung!

3) Nagel's Handbuch der Physiologie Bd. 4 S. 527. 1907. 
darüber auch nie im unklaren geblieben --, dass, wenn ich etwa die Kette:

$\mathrm{Hg}, \mathrm{HgCl} \mid$ Ringer | Muskel | LiCl | Ringer | $\mathrm{HgCl}, \mathrm{Hg}$ aufbaue, die gemessene E.-M.-K. nicht ganz allein die E-M.-K. des Muskels zu repräsentieren braucht, weil die Summe der Potentialdifferenzen: Ringer | Interfibrillärflüssigkeit, Interfibrillärflüssigkeit | $\mathrm{LiCl}$ und $\mathrm{LiCl}$ | Ringer nicht ohne weiteres gleich Null gesetzt werden darf. Dieser Fehler meiner Messungen, der sich übrigens kaum vermeiden lassen wird, falls man sämtliche Alkalisalze prüfen will, ist indessen für die Beurteilung der Versuche und vor allem auch für die aus ihnen gezogenen Schlüsse völlig irrelevant. Denn die Ruhestromwerte spiegeln, wie ich schon in meiner früheren $\mathrm{Ab}$ bandlung gezeigt babe ${ }^{1}$ ), auf das deutlichste die Erregbarkeitsabstufungen wider, welche an den Muskeln mit denselben Salzlösungen zu erzeugen sind, die auch das Auftreten der Ruheströme herbeiführen. Um das zu zeigen, gebe ich zum Vergleich mit der Tabelle für die E.-M.-K.-Werte eine Tabelle für die Rückkehr der Erregbarkeit von Rohrzucker-Muskeln in den verschiedenen Salzlösungen nach den Versuchen von Overton, ergänzt durch einige eigene Beobachtungen:

\begin{tabular}{|c|c|c|c|c|c|}
\hline & $\mathrm{K}$ & $\mathrm{Rb}$ & Cs & $\mathrm{Na}$ & $\mathrm{Li}$ \\
\hline 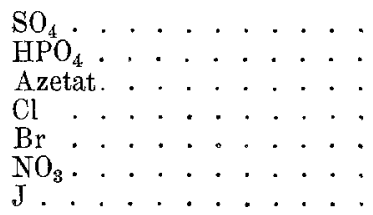 & $\begin{array}{l}- \\
\overline{-} \\
\overline{-} \\
\overline{-}\end{array}$ & - & - & $\begin{array}{l}+ \\
+ \\
+ \\
+ \\
+ \\
+\end{array}$ & $\begin{array}{l}+ \\
+ \\
+ \\
+\end{array}$ \\
\hline
\end{tabular}

Minuszeichen bedeuten, dass durch das betreffende Salz die Erregharkeit nicht wieder hergestellt werden kann, Pluszeichen bedeuten, dass sie zurückkehrt. Man sieht, dass alle Salze, welche die Muskelsubstanz "negativ" machen, unfähig sind, die Erregbarkeit zu restituieren, während alle Salze, welche "elektroneutral" sind oder positivieren, wiedererregbar machen können.

Dieser von mir bemerkte Zusammenhang, welchen v. Frey

1) Pflüger's Arch. Bd. 106 s. 629. 1905. 
bei seiner Kritik ganz ausser acht gelassen hat, kann natürlich kein blosser Zufall sein, vielmehr ist er gerade ein Beweis dafür, dass meine Strommessungen im grossen ganzen richtig sind. Denn dieser Zusammenbang muss ja bei einwandsfreien Messungen von vornherein erwartet werden, da "Negativierung" oder nach Hering's Ausdruck "absteigende Änderung" den Zustand des Erregtseins charakterisiert, oder anders ausgedrückt: da erregbar eine solche lebende Substanz ist, die noch elektroneutral oder positiv, also noch negativierbar ist.

Dazu sei noch hervorgehoben, dass Schwarz vor kurzem ${ }^{1}$ ) gezeigt hat, dass die Erregbarkeit von Rohrzucker-Muskeln durch die verschiedenen Natriumsalze verschied en gut restituiert wird, am besten durch Rhodanid und Jodid, dann durch Bromid, Nitrat, Chlorid, Azetat, Sulfat in absteigender Reihe. Diese Anordnung stimmt auf's vortrefflichste zu meinen Rubestrom-Werten, bildet also einen neuen Beweis für deren Richtigkeit. -

v. Frey hat meine Versuche noch weiter kritisiert; nach dem vorher zitierten Satz fährt er fort: „Dazu kommt, dass für den Muskel giftige und harmlose Lösungen unterschjedslos verwendet werden und Mitteilungen fehlen, ob der Muskel noch erregbar, reversibel gelähmt oder abgestorben war. Unter diesen Umständen jst es nicht möglich, zu den sehr weit gehenden Schlussfolgerungen des Verfassers Stellung zu nehmen."

Auch diese Kritik verfehlt ihr Ziel. Wie Overton ${ }^{2}$ ) gezeigt hat, gibt es unter den Alkalisalzen in der Tat solche, welche reversibel lähmen, also eventuell ganz harmlos sind, nämlich wenn sie wieder entfernt werden, und solche, welche irreversibel lähmen können, also giftig sind. Unter den Kalisalzen gehören z. B. zu den ersten das Sulfat, Tartrat, Phosphat, zu den zweiten das Chlorid, Bromid, Nitrat, Jodid; in ähnlicher Weise verteilen sich die Rubidiumund Ammonsalze je nach dem Anion in die Klasse der harmlosen und der giftigen. Nun hebt aber Overton extra hervor ${ }^{3}$ ), dass die Giftigkeit der Chloride, Bromide usw. nur bei längerer Einwirkung zur Geltung kommt, während eine Einwirkung der reinen isotonischen Lösungen für 10--12 Minuten nichts schadet. Dem vollkommen entsprechend haben mich meine Versuche belehrt, dass

1) Pflüger's Arch. Bd. 117 S. 161. 1907.

2) Pflüger's Arch. Bd, 105 S. 176. 1904.

3) 1. c. S. 188. 
die Erscheinung des durch die Salzlösungen erzeugten Ruhestromes im allgemeinen durchaus reversibel ist, wenn die Salze nicht länger als 10 Minuten gewirkt haben, und dementsprechend beziehen sich auch, wie ich besonders hervorhob, meine Angaben über die Grösse der E.-M.-K. auf 10 Minuten lange Einwirkung ${ }^{1}$ ). Als Beweis der Reversibilität des Effektes einer reinen isotonischen KCl-Lösung von selbst 15 Minuten Wirkungsdauer greife ich folgendes Protokoll vom 10. Juni 1904 heraus:

\begin{tabular}{|c|c|c|c|c|c|}
\hline Muskelende taucht in Ringer: & gh 15 , & $9 \times 25^{\prime}$ & 9 h. 34 ' & 9 h $40^{\prime}$ & \\
\hline Nillivolt: & 6,0 & 6,5 & 7,25 & 8,25 & \\
\hline Dann in $0,83 \% \mathrm{KCl}:$ & $9 \mathrm{k} 42^{\prime}$ & 9 h $46^{\prime}$ & $9 \mathrm{~h} 50^{\prime}$ & 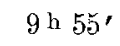 & \\
\hline Millivolt: & 16,0 & 26,0 & 30,5 & 32,, $\mathbf{3}$ & \\
\hline Dann wieder in Ringer: & $\begin{array}{c}9 \text { h } 57^{r} \\
19,0\end{array}$ & $\begin{array}{c}10 \mathrm{~b} 8^{\prime} \\
16,0\end{array}$ & $\begin{array}{c}10 \text { h } 18^{\prime} \\
13,5\end{array}$ & $\begin{array}{c}10^{\mathrm{h}} 29^{\prime} \\
10,75\end{array}$ & $\begin{array}{c}10^{h} 41^{\prime} \\
8, \mathbf{5}\end{array}$ \\
\hline
\end{tabular}

Die Salzlösungen waren also bei meiner Art der Verwendung, entgegen v. Frey's Annahme, samt und sonders harmlos.

Aber selbst wenn das nicht der Fall gewesen wäre, so balte ich die Unterscheidung der harmlosen und der schädigenden Salzlösungen schon deshalb für gleichgültig, weil dieser Unterschied, wie die Tabelle auf Seite 2 lehrt, in der elektrischen Reaktion, im Rubestrom, sich gar nicht geltend macht, während die Hauptsache, der Parallelismus mit der Erregbarkeit (Tabelle S. 496) sehr wohl zum Ausdruck kommt. Damit erweist sich die tatsächlich vorhandene Giftigkeit eines Teiles der Salze bei längerer Einwirkung als etwas Sekundäres, relativ Belangloses. Für diese Giftigkeit habe ich übrigens eine Erklärung zu geben versucht ${ }^{2}$ ).

Ich komme also zu dem Schluss, dass die von mir gemessenen E.-M.-K. der durch isotonische Salzlösungen erzeugten reversiblen Ruheströme die durch dieselbensalzlosungenerzeugten reversiblen Abstufungen in der Erregbarkeit getreu widerspiegeln, und aus diesem Parallelismus leite ich die Berechtigung zu den in der Tat weitgehenden, von mir gezogenen Konsequenzen her, welche v. Frey beanstandet hat.

1) Pflüger's Arch. Bd. 106 S. 610 und 611.

2) Physikal. Chemie der Zelle und der Gewebe, 2. Aufl., S. 274 und 278. 1906. 
Beiträge zur physikalischen Chemie der Erregung und der Narkose. 499

\section{Der Erregungsvorgang als Kolloidprozess.}

Diese Konsequenzen waren folgende: Die Befunde von Overton und Schwarz über die Abstufbarkeit der Erregbarkeit durch die verschiedenen Salze wären ebenso eine Summe seltsamer, ganz zusammenhangloser Fakten, wie die verschiedenen von mir gemessenen Ruhestromwerte es wären ohne meinen Hinweis auf die Beziehungen zwischen Kolloiden und Salzen. Die Existenz dieser Beziehungen führt aber unmittelbar zu der Hypothese, dass, g e rade sowiedie elektrischeReaktion a f die Salzeinwirkung, auch die Reaktion der Erregbarkeitsänderung a uf die Salzeinwirkung einer Änderung in der Konsistenz oder dem Lösungszustand der Protoplasmakolloide zuzuschreiben ist. Diese Annahme habe ich inzwischen durch den Nachweis gestützt ${ }^{1}$ ), dass beim Nerven sich der durch ein Salz erzeugte Erregbarkeitsgrad im Aussehen der Achsenzylinder, die doch wesentlich aus Kolloiden bestehen, dokumentiert.

Die Ruhestrom-Messungen führen aber noch $\mathrm{zu}$ einer weiteren Hypothese: wenn man etwa durch ein Kalisalz eine Muskelstelle negativ macht und gleich darauf durch Auswaschen mit RingerLösung die Negativität wieder beseitigt, so ist dieser ganze Vorgang einigermaassen eine Nachahmung der Verhältnisse bei der normalen Erregung, bei der auch lokal eine Negativität entsteht und gleich darauf wieder verschwindet. Führt man nun die durch das Kalisalz erzeugte Negativität, so wie ich es tue, auf eine mit Veränderung der Ionenpermeabilität der Plasmahaut einhergehende Kolloidzustandsänderung zurück, so kommt man zu der Vermutung, dass die die Erregung charakterisierende wellenförmig sich ausbreitende Negativitätswelle des Aktionsstromes von einerwellenförmig über das erregbare Gebilde, Muskel oder Nerv, hinlaufenden Kolloidzustandsänderung herrührt, welcher selbst wieder eine wellenförmige, etwa vom Freiwerden von K-Ionen begleitete chemische Reaktion zugrunde liegt.

Diese neue Hypothese lässt sich folgendermaassen prüfen: wenn es Mittel gibt, die Protoplasma-Kolloide gegen die Kinwirkung der Ionen unempfindlich zu machen, so muss nun auch der elektrische 
Ausdruck dieser Einwirkung in Fortfall kommen. Und: wenn der Kolloidprozess eine regelmässige und notwendige Komponente des Erregungsvorgangs darstellt, dann muss seine Sistierung auch die Erregbarkeit aufheben.

Die Narkotika sind nun solche Mittel, welche die Zustandsänderung der Protoplasma-Kolloide und damit den Erregungsvorgang hemmen.

\section{Einwirkung der Narkotika auf den Ruhestrom.}

Dass durch Narkose der elektrische Ausdruck der Erregung, der Aktionsstrom, in Fortfall gebracht wird, ist eine bekannte und öfter konstatierte Tatsache ${ }^{1}$ ). Ich behaupte nun, dass dies mit dem Wegfall der präsumptiven Kolloidkomponente des Erregungvorganges zusammenhängt. Vor allem spricht dafür, dass das Auftreten der Salzruheströme, welehes, wie wir sahen, kaum anders dennalsein Effekt auf die Plasmahaut-Kolloide gedeutet werden kann, durch die Narkotika gehemmt wird.

Hierüber war bisher fast nichts bekannt; die einzige Angabe von $B$ iederman $\mathbf{n}^{2}$ ), dem Entdecker der Salzströme, dass „lokale Behandlung mit Kalisalzen auch den Äthermuskel an der betreffenden Stelle negativ macht", besagt das gerade Gegenteil von dem eben aufgestellten Satz.

Meine Versuche sind wiederum an Sartorien von Fröschen (vorzugsweise Temporarien) ausgeführt ${ }^{3}$ ).

Zunächst wurden passende Narkotika und deren geeignete Konzentrationen ausprobiert, dabei wurden wenig flüchtige Mittel bevorzugt. Das Ausprobieren geschah auf zweierlei Weise; erstens wurden Muskeln in versehieden konzentrierte Lösungen eines Narkotikums eingelegt, mit dem Induktionsapparat der Abfall der Erregbarkeit verfolgt, und vor allem darauf geachtet, $o b$ dann in einer Ringerlösung die ursprüngliche Erregbarkeit zurückkehrt. Zweitens wurde das Ende eines unversehrten Sartorius in Ringerlösung gehängt,

1) Siehe dazu etwa: Boruttau, Pflüger's Arch. Bd. 68 S. 351.1897 und Wedensky, Pflüger's Arch. Bd. 82 S. 134. 1900.

2) Sitzungsber. d. Wiener Akad. Bd. 97 Heft III S. 84. 1888.

3) Kurze Mitteilung derselben siehe: Vierteljahrschrift der Naturforsch. Gesellsch. Zürich, April 1907. Die Versuche sind zum Teil in Gemeinschaft mit Privatdozent Dr. Schatil off (Charkow) ausgeführt. 
aus dieser und vom Muskel selbst mit Ringer-Calomel-Elektroden zum Galvanometer abgeleitet, eine Zeitlang beobachtet, und dann in Ringer-Lösung mit Narkotikum-Zusatz übertragen und beobachtet, ob der Muskel stromlos bleibt resp. ob das bisherige elektrische Verhalten keine wesentliche Änderung erleidet. Der Verlauf eines solchen Versuchs mit Chloralhydrat war z. B. folgender:

\begin{tabular}{|c|c|c|c|c|c|c|c|}
\hline Zeit & $\begin{array}{c}\text { Milli- } \\
\text { volt }\end{array}$ & \multicolumn{2}{|c|}{ Muskel hängt in: } & Zeit & $\begin{array}{c}\text { Milli- } \\
\text { volt }\end{array}$ & \multicolumn{2}{|c|}{ Muskel hängt in: } \\
\hline $\begin{array}{rr}4 & 35 \\
4 & 45 \\
4 & 55 \\
5 & 5 \\
5 & 15 \\
5 & 25 \\
5 & 35 \\
5 & 45 \\
5 & 55\end{array}$ & $\begin{array}{l}+2,8 \\
+2,5 \\
+2,2 \\
+1,4 \\
+1,0 \\
+0,7 \\
+0 \\
+0 \\
-0,5\end{array}$ & $\begin{array}{l}\text { Ringer } \\
\begin{aligned} & \\
" & \\
" & +0,3 \% \\
" & +0,3 \% \\
" & +0,3 \% \\
" & +0,6 \% \\
" & +0,6 \% \\
" & +0,6 \%\end{aligned}\end{array}$ & $\begin{array}{c}\text { Chloralh. } \\
\text { " } \\
" \\
" \\
"\end{array}$ & $\left|\begin{array}{rr}6 & 05 \\
6 & 15 \\
6 & 25 \\
6 & 35 \\
6 & 45 \\
6 & 55 \\
7 & 5 \\
7 & 15\end{array}\right|$ & $\begin{array}{l}+3,4 \\
+11,5 \\
+19,3 \\
+25,0 \\
+27,0 \\
+27,5 \\
+28,5 \\
+28,7\end{array}$ & $\begin{array}{r}\text { Ringer }+1,2 \% \\
"+1,2 \% \\
" \quad+1,2 \% \\
"+1,2 \% \\
" \quad+1,2 \% \\
" \quad+1,2 \% \\
" \quad+1,2 \% \\
" \quad+1,2 \%\end{array}$ & $\begin{array}{c}\text { Chloralh. } \\
\text { " } \\
" \\
" \\
" \\
" \\
"\end{array}$ \\
\hline
\end{tabular}

Es zeigt sich, dass bis zu $0,6 \%$ das Chloralhydrat indifferent ist, dass es aber bei grösserer Konzentration stark negativiert. In dieser Weise wurden für verschiedene Narkotika Schwellenkonzentrationen ermittelt. Bei der Nachprüfung mit der ersten Methode ergab sich, dass bei den betreffenden elektrisch-indifferenten Konzentrationen die Muskelerregbarkeit noch keineswegs vollständig erlischt. Grössere, total lähmende Konzentrationen liessen sich aber nicht verwenden, eben weil Negativierung, und zwar irreversible Negativierung eintritt, die sich übrigens bald auch äusserlich durch Einschrumpfen und Trübung der Muskulatur dokumentiert.

Am vorteilhaftesten von allen geprüften Substanzen erwies sich das von Overton ${ }^{1}$ ) empfohlene Phenyl-Urethan; bei einer Konzentration von $0,04-0,05 \%$ bleiben die Muskeln lange am Leben und erholen sich in reiner Ringer-Lösung vollständig. Nächst dem Phenyl-Urethan fand ich das Äthyl-Urethan in einer Konzentration von $2 \%$ besonders geeignet.

Der Einfluss der Narkotika auf die Ruhestromentwicklung dureh die isotonischen Salzlösungen wurde nun in folgender Weise untersucht: rechter und linker Sartorius eines kurarisierten Frosches wurden gleichzeitig verwendet; der eine kam für 1/2 Stunde in Ringer-Lösung, der andere ebensolange in Ringer plus Narkotikum, also z. B. in

1) Studien über Narkose S. 115. Jena 1901. 
Ringer-Lösung mit einem Zusatz von 0,04\% Phenyl-Urethan. Dann wurden beide Muskeln in feuchte Kammern gehängt und ihre Enden in die Lösungen eingetaucht, in denen sie bisher gelegen hatten. Aus den Lösungen sowie von den Muskelmitten wurde mit RingerCalomel-Elektroden abgeleitet und nun zirka eine Stunde lang der Gang der E.-M.-K. beobachtet. Dann wurden die beiden Lösungen ausgewechselt gegen solche, in denen statt der Ringer-Salze ein elektrisch indifferentes Salz enthalten war, also z. B. gegen $0,95 \% \mathrm{NaNO}_{3}$ und $0,95 \% \mathrm{NaNO}_{3}+0,04 \%$ Phenyl-Urethan, und die Änderungen der E.-M.K. weiter beobachtet. In der Art wurde eine grosse Zahl von Salzen, sowohl negativierenden als auch positivierenden, durchprobiert.

Negativierende Salze: Ich beginne die Mitteilung der Resultate mit diesen, weil sie die wichtigeren sind, da ja auch normaler Weise bei der Erregung Negativierung zustandekommt. Einen Einfluss der Narkotika auf die Stromentwicklung bekommt man nicht deutlich $z u$ seben, wenn man die reinen isotonischen Lösungen der positivierenden Salze verwendet, wie es z. B. Biedermann machte. Vielmehr muss man die Salze in geringen Konzentrationen anwenden, und das beste ist es nach meinen Erfahrungen, wenn man womöglich die positivierende Wirkung durch die negativierende Wirkung eines in geeigneter Konzentration zugesetzten zweiten Salzes abschwächt. Wenn man so verfährt, dann lässt sich zeigen, dass durch die Narkotika stets die stromentwickelnden Fähigkeiten der Alkalisalze gehemmt werden.

Ich gebe einige Beispiele in Kurvenform; auf der Abszisse sind die E.-M.-K. in Millivolt, auf der Ordinate die Zeiten abgetragen: (Siehe Fig. 1, 2, 3 und 4.)

In allen vier Versuchen wurde also neben dem negativierenden $\mathrm{K}$ - oder Rb-Salz zur genügenden Abstumpfung von deren Wirkung das an und für sich positivierende $\mathrm{NaNO}_{3}$ verwendet.

Man sieht nun an den Kurven aufs deutlichste den Einfluss des Narkotikums. Während ohne dasselbe die E.-M.-K. sofort und rasch ansteigt, wenn das Muskelende dem negativierenden Salz ausgesetzt wird, wird diese Wirkung annuliert, eingeschränkt oder wenigstens verzögert, wenn der Muskel narkotisiert ist. Also im Sinne meiner Hypothese kann man sagen: durch Narkotisierung wird ebensogut der Einfluss der Ionen auf die Plasmahaut-Kolloide von aussen 
Beiträge zur physikalischen Chemie der Erregung und der Narkose. 503 her gehemmt, so dass der elektrische Effekt dieses Einflusses beschränkt wird, wie bei der normalen. Erregung der Einfluss der

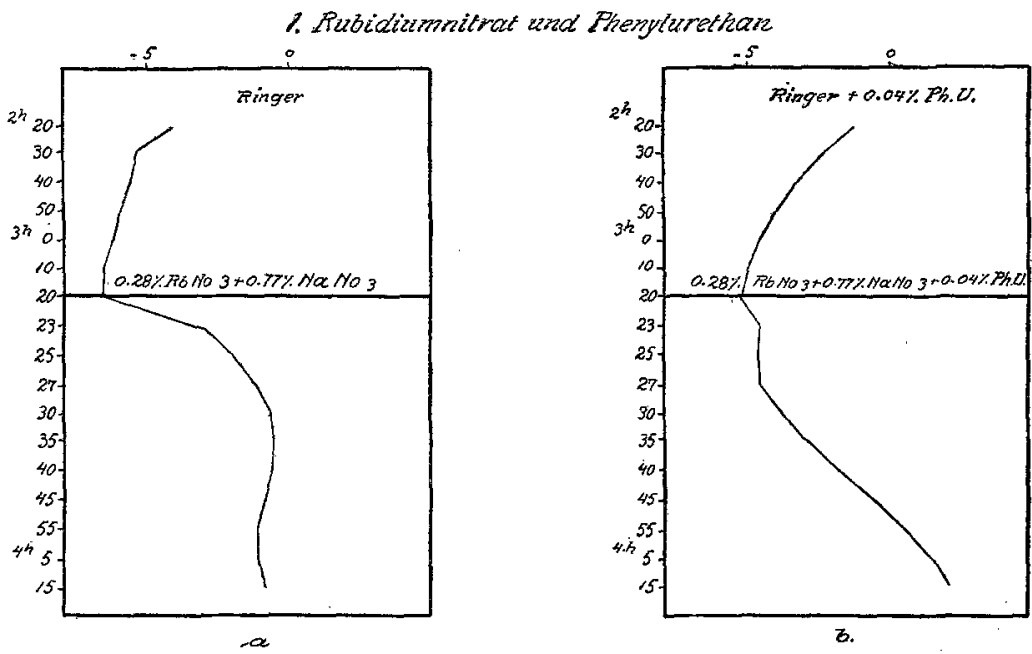

Fig. 1.

2. Rubiatumnitrat una' Phenyturethan
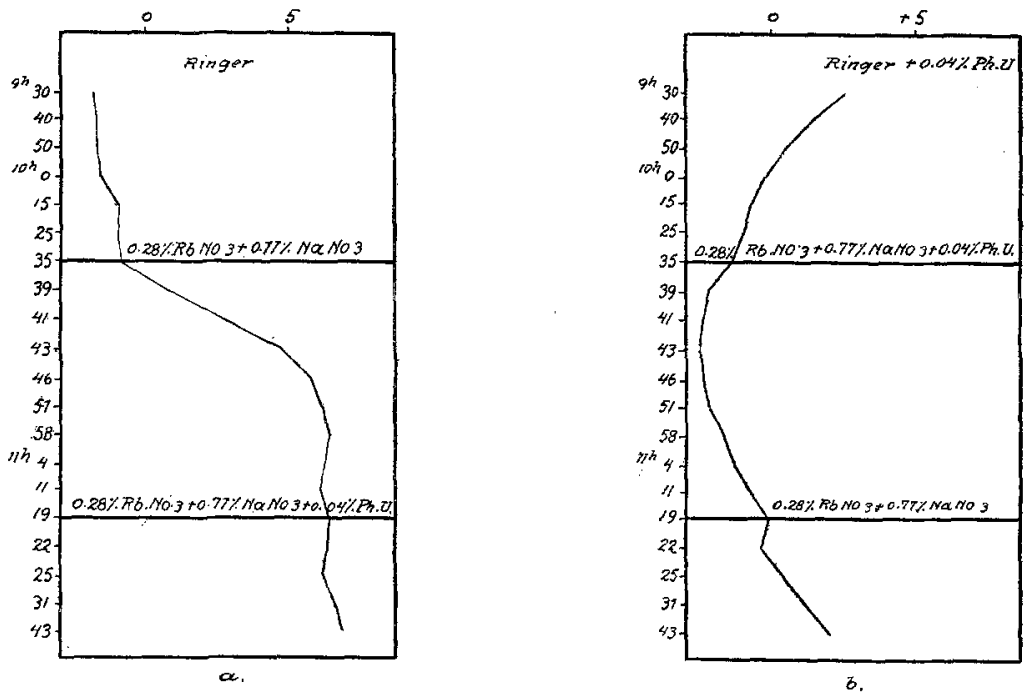

Fig. 2.

in neren Elektrolyte auf die Kolloide gehemmt wird, so dass der Aktionsstrom nicht mehr zustande kommt. 
Ganz ähnlich verliefen Versuche mit den negativierenden Salzen: $\mathrm{KCl}, \mathrm{RbCl}, \mathrm{NH}_{4} \mathrm{Cl}, \mathrm{NH}_{4} \mathrm{NO}_{3}, \mathrm{Cs}_{2} \mathrm{SO}_{4}$. Einen Versuch mit $\mathrm{KCl}$ gebe

3. Hatiummitrat.a. Phenyturetran
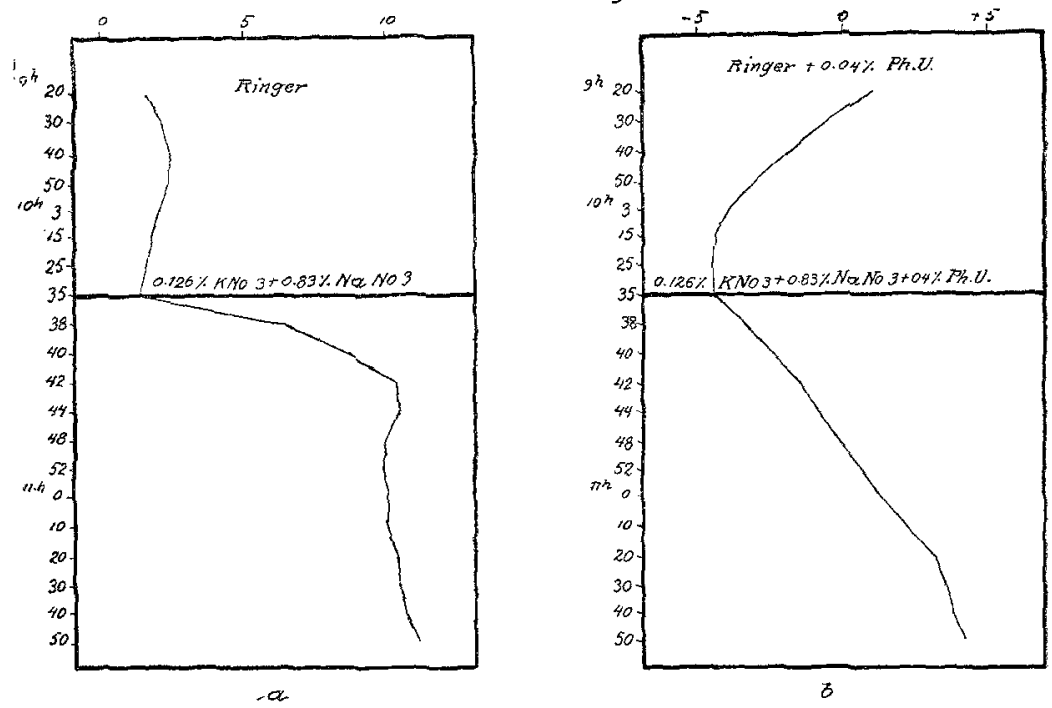

Fig. 3.

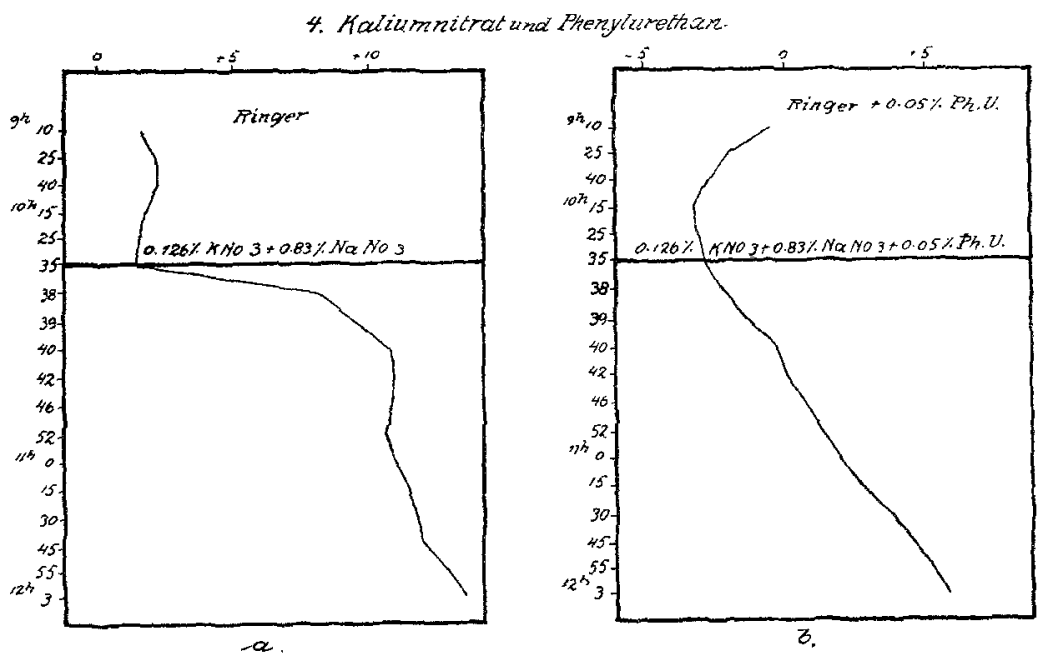

Fig. 4.

ich noch in Kurvenform wieder, um zu zeigen, wie sich der Einfluss des Narkotikums auch danu äussert, wenn kein kompensierendes positivierendes Salz mit verwendet wird. (Siehe Fig. 5.) 
Beiträge zur physikalischen Chemie der Erregnng und der Narkose. 505

Positivierende Salze. Von positivierenden Salzen wurden untersucht: $\mathrm{LiCl}, \mathrm{LiBr}, \mathrm{NaNO}_{3}$, NaJ. Da die stromentwickelnde Fähigkeit dieser Salze auch in reiner isotonischer Lösung relativ

5 Kalium chlarid und Phenylurethan
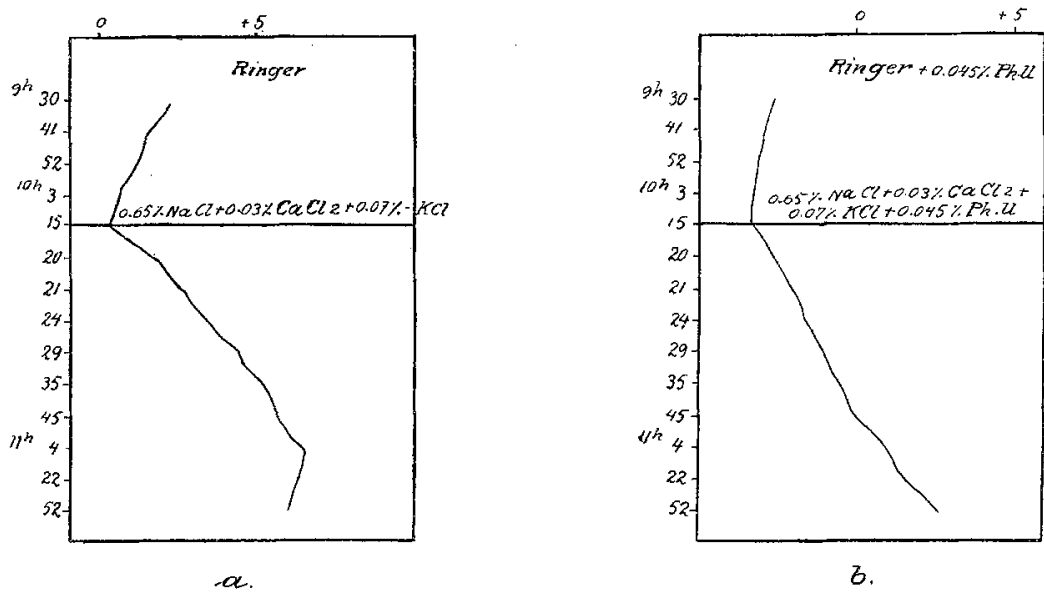

Fig. 5.

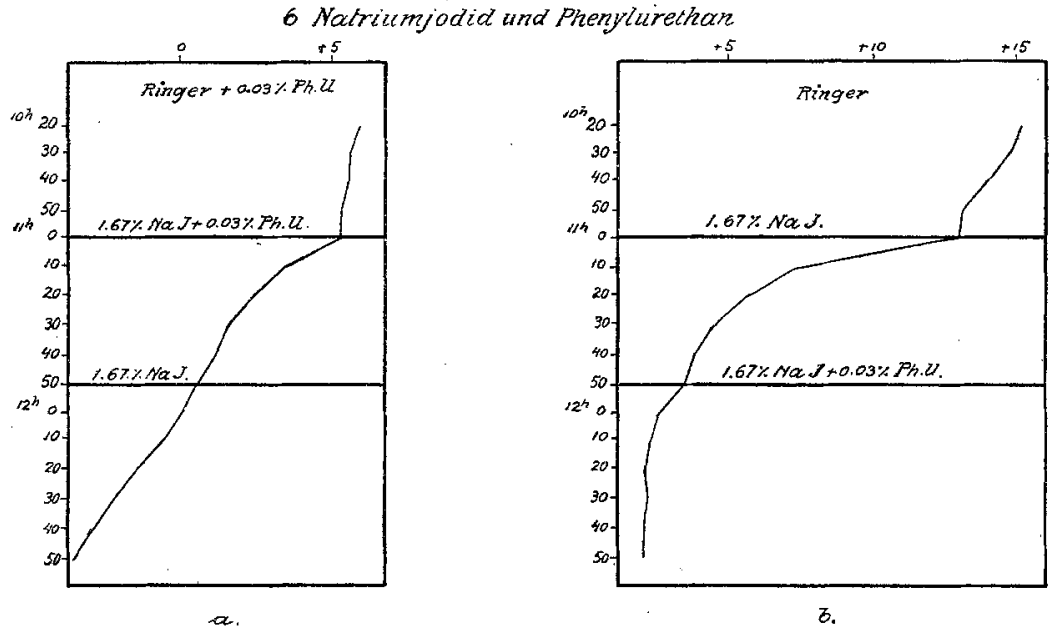

Fig. 6 .

schwach ist ${ }^{1}$ ), so konnte von eiper partiellen Kompensation mit einem negativierenden Salz hier abgesehen werden. Als Beispiel gebe ich einen der Versuche mit NaJ wieder. (Siehe Fig. 6.)

1) Siehe Pflüger's Arch. Bd. 106 S. 610. 1905. 
Verschiedene Narkotika. In den bisher mitgeteilten Versuchen kam allein Phenylurethan zur Verwendung. Ausser mit diesem wurden mit demselben Erfolg Versuche mit Äthylurethan, Chloralhydrat, Chlor of orm und Hypnon ausgeführt. Als Beispiel für deren Wirkung gebe ich folgende Versuche mit Chloralhydrat und mit Chloroform wieder. (Siehe Fig. 7 und 8.)

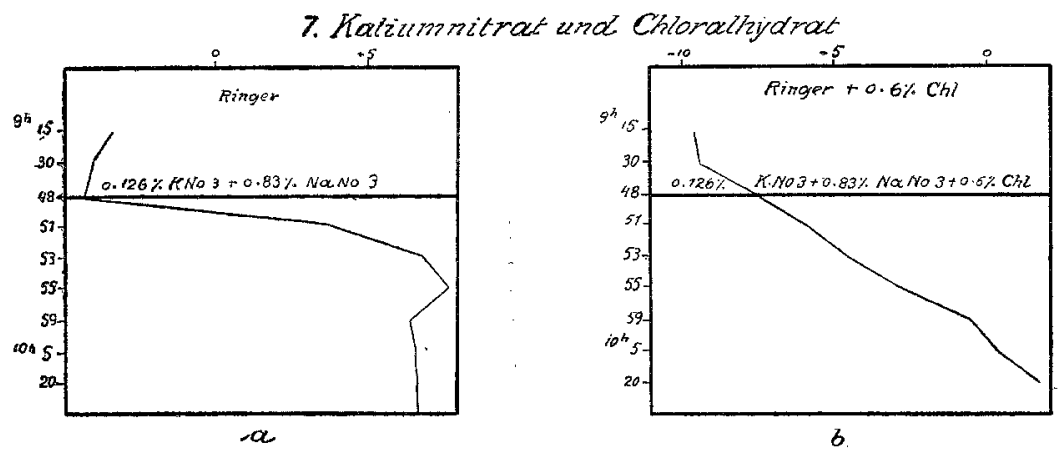

Fig. 7.
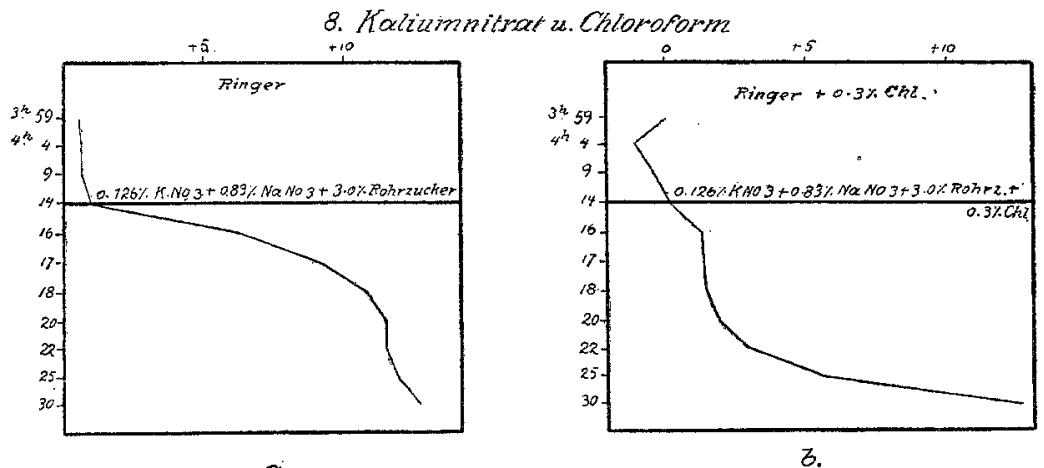

Fig. 8.

In dem Chloroformversuch (Nr. $8 \mathrm{~b}$ ) trat bald nach Einwirkung des Narkotikums Absterben des Muskels ein; dies äussert sich in dem raschen Anstieg der Kurve.

Im übrigen sieht man, dass Chloralhydrat und Chloroform, sowie auch die anderen genannten Narkotika, gerade so wirken wie Phenylurethan.

Einfluss organischer Nichtnarkotika. Es bleibt nun noch zu fragen, ob wir es hier mit einer für die Narkose spezifischen Wirkung der verwendeten Mittel zu tun haben, und ob nicht viel- 
Beiträge zur physikalischen Chemie der Erregung und der Narkose. 507

leicht allen möglichen organisehen Verbindungen der gleiche Einfluss auf den Salzruhestrom zukommt. Zu diesem Zweck wurde die Wirkung der Narkotika mit derjenigen von Rohrzucker,

\section{Tratiumnitrat u. Traubenzucker.}
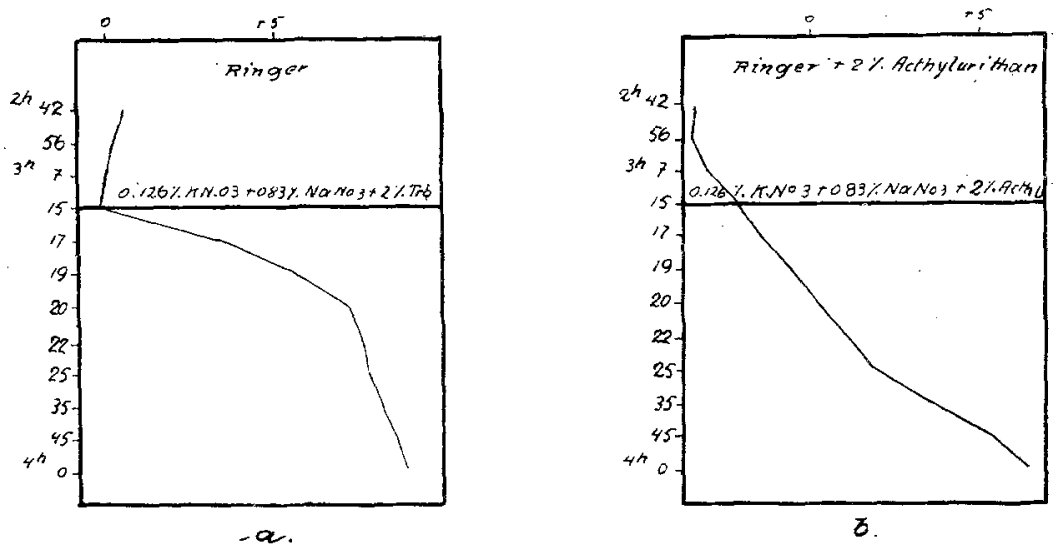

Fig. 9.

10. Kaliumnitratu. Hornstoff
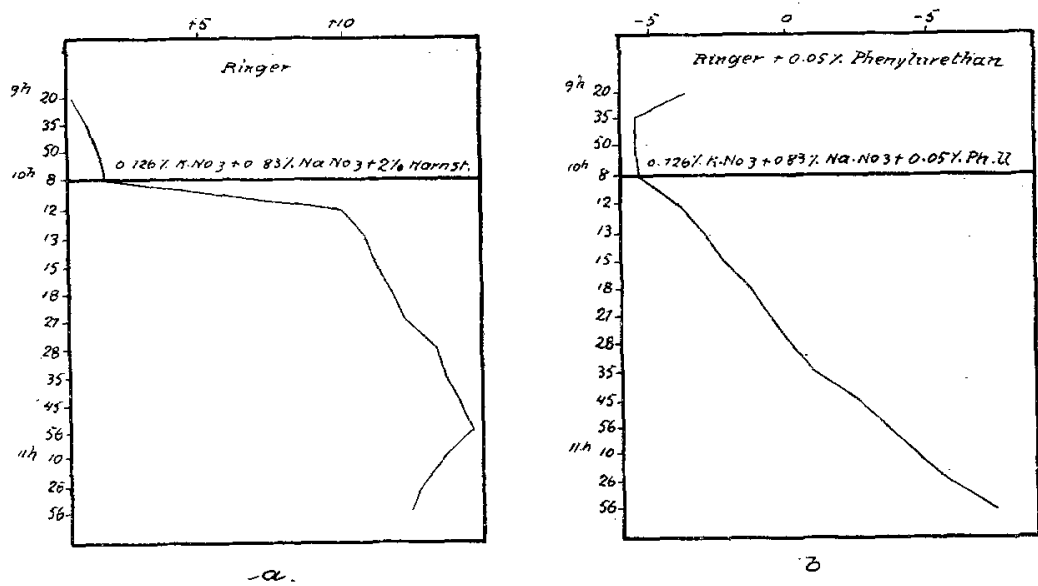

Fig. 10.

Traubenzucker, Mannit und Harnstoff verglichen. Es zeigte sich, dass der Zusatz der letzteren zu der Lösung eines negativierenden Salzes dessen ruhestromentwickelnde Eigenschaften gar nicht stört. Die Hemmung des Salzrubestromes ist also eine spezifische Wirkung der Narkotika. 
Ein Beispiel für die Indifferenz des Rohrzuckers ist schon in Versuch 8 gegeben. Weitere Beispiele für Traubenzucker und Harnstoff folgen. (Siehe Fig. 9 u. 10.)

Hiernach ist nun also zusammenfassend über den Erregungsvorgang folgendes zu sagen: die früher erörterten Versuche liessen es als sehr wahrscheinlich erscheinen, dass die die Erregung charakterisierenden Aktionsströme ebenso wie die Salzruheströme auf einen Kolloidprozess zurückzuführen sind. Ist das richtig, so ist es auch denkbar, dass die Salzruheströme gerade so durch die Narkotika gehemmt werden können wie die Aktionsströme. Das triff, wie die Versuche zeigen, tatsächlich zu. Also ist der Schluss zu ziehen, dass die Narkotika an den Plasmahaut-Kolloiden angreifen und deren zur Erregung gebörige Zustandsänderung hemmen.

\section{Das mikroskopische Bild der Narkose.}

Als nächste Aufgabe ergibt sich alsdann der Versuch, die Hemmung des Kolloidvorgangs durch die Narkotika direkt nachzuweisen. Diesen Versuch habe ich an Ischiadici vom Frosch auszuführen begonnen.

Es hatte sich früher ergeben ${ }^{1}$ ), dass, wenn man Nervenfasern, welche zuvor etwa durch Kaliumsulfat gelähmt worden sind, nach Bethe primär färbt, die Achsenzylinder derselben ein anderes Bild gewähren, als wenn die Nervenfasern zuvor mit Natriumsulfat oder Lithiumsulfat behandelt worden sind, in denen die Erregbarkeit erhalten bleibt. Im ersten Fall sind die Achsenzylinder breit und (durch Toluidinblau) matt gefärbt, im zweiten sind sie schmal und intensiv gefärbt. Der Ausdruck des elektronegativen Verhaltens, des Erregtseins (s. Seite 497) wäre danach eine Auflockerung des Achsenzylinders resp. eine Auflockerung seiner Fibrillen, der Ausdruck der Elektroneutralität, des Erregbarseins, eine relativ dichte Struktur des Achsenzylinders.

Wenn nun die vorher ausgesprochene Ansicht von der lähmenden Wirkung der Narkotika richtig ist, so ist zu erwarten, dass an einem narkotisierten Nerven die Auflockerung der Achsenzylinder

1) Höb er, Zentralblatt f. Physiologie Bd. 19 S. 390.1905. 
durch Kaliumsulfat oder ein ähnliches negativierendes Salz nicht mehr zustande kommt. Das scheint nun in der Tat der Fall zu sein.

Meine Beobachtungen in dieser Hinsicht erstrecken sich allerdings bis jetzt nur auf ein einziges Salz und ein einziges Narkotikum, bedürfen also noch sehr der Vervollständigung; dennoch teile ich sie hier bereits mit, weil sie durchaus zugunsten der aufgestellten Theorie sprechen. Als Narkotikum wurde Äthylurethan in einer Konzentration von $2 \%$ verwendet, nachdem ich mich davon überzeugt hatte, dass der Nerv, der darin innerhalb einiger Stunden seine Erregbarkeit zwar nicht ganz verliert, aber doch bis etwa auf $1 / 100$ einbüsst, nach Übertragung in eine narkotikumfreie Lösung wieder fast die ursprüngliche Erregbarkeit zurückgewinnen kann. Als Salz wurde Kaliumsulfat benutzt, dessen lähmende Wirkung in isotonischer Lösung ebenfalls reversibel ist (s. S. 497). Im übrigen wurden die Versuche folgendermaassen ausgeführt: der eine mit dem Gastrocnemius in Verbindung gebliebene Ischiadicus einer Esculenta wurde bis an den Muskel in eine mit Eis gekühlte Lösung von $1,21 \% \mathrm{Na}_{2} \mathrm{SO}_{4}+0,03 \% \mathrm{CaCl}_{2}$ gehängt, der andere in die gleiche Lösung mit einem Zusatz von $2 \%$ Äthylurethan ${ }^{1}$ ). Nach ca. 2 Stunden wurden, wenn sich zeigte, dass der erste Nerv und der zweite Muskel ihre volle Erregbarkeit behalten hatten, die beiden Nerven in die entsprechenden Lösungen mit $1,48 \% \mathrm{~K}_{2} \mathrm{SO}_{4}$ übertragen. Hier blieben die Nerven ca. 12 Stunden und verloren ihre Erregbarkeit vollkommen (während die Muskelerregbarkeit erhalten blieb) ${ }^{2}$ ). Dann wurde je ein Stück der beiden Nerven auf demselben Objektträger zerzupft und nach $B$ ethe primär gefärbt. Es zeigte sich, dass die Achsenzylinder der beiden Nerven gerade so verschieden aussahen, wie etwa ein $\mathrm{Na}_{2} \mathrm{SO}_{4}$ - und ein $\mathrm{K}_{2} \mathrm{SO}_{4}$-Nerv, d. h. unter dem Einfluss des Narkotikums bleibt die Auflockerung der Achsenzylinder durch Kaliumsulfat aus, welche ohue das Narkotikum zustande kommt; der narkotisierte Nerv sieht also aus wie ein erregbarer, negativierbarer, obgleich er dem negativierenden Salz ausgesetzt war. Das so gewonnene mikroskopische Bild der Narkose verifiziert demnach die Theorie. -

1) Der $\mathrm{CaCl}_{2}$-Zusatz geschah, um das Erlöschen der indirekten Muskelerregbarkeit sicher zu verhindern (siehe Locke, Journ. of physiol. vol. 18 p. 318. 1895 u. Pflüger's Arch. Bd. 54 S. 501. 1893.)

2) Heruntersetzen liess sich diese Zeitdauer bisher nicht, ohne das Resultat zu gefährden. 
Es ist nun bekanntlich schon einmal auf mikroskopisch erkennbare Eigenschaften des narkotisierten Nerven von Bethe ${ }^{1}$ ) hingewiesen worden, und es fragt sich, wie sich meine Beobachtungen zu denen Bethe's verhalten. Bethe hat gefunden, dass die durch einen konstanten Strom an der Kathode erzeugte Steigerung der Erregbarkeit sich in einer stärkeren Färbbarkeit der Neurofibrillen äussert, und dass die an der Anode sich einstellende Erregbarkeitsherabsetzung sowie überhaupt jede irgendwie zustande gebrachte Verminderung der Erregbarkeit in einer abgeschwächten bis aufgehobenen Färbbarkeit zum Ausdruck kommt. Diese Bilder des Katund Anelektrotonus treten nicht mehr auf bei der Durchströmung des narkotisierten Nerven, sondern da bleiben die Fibrillen gleichmässig färbbar und bieten das Bild des normalen erregbaren Nerven. Bethe gibt den Tatsachen (im Zusammenhang mit anderen von ihm beobachteten Erscheinungen) etwa folgende Deutung: der funktionsfähige Nerv ist ausgezeichnet durch das Vorhandensein einer lockeren Bindung der (färbbaren) "Fibrillensäure“ an die Neurofibrillen. Der konstante Strom hebt diese Bindung an der Anode auf und transportiert die Fibrillensäure (kataphoretisch) kathodenwärts; die Folge ist Schwund der Erregbarkeit und Leitfähigkeit an der Anode, da der normalen Nervenerregung und der Fortleitung der Erregung ein Wechsel der Affinität zwischen Fibrille und Fibrillensäure und eine Verschiebung der letzteren zugrunde liegt. An der Kathode wird hingegen die Bindung verstärkt, weitere Fibrillensäure wird von der Anode her durch den Strom herantransportiert und die Erregbarkeit steigt. Die Narkotika haben nun die Eigenschaft, entweder die zur Erregung notwendige Zersetzbarkeit der Verbindung zwischen. Fibrille und Fibrillensäure oder die zur Fortleitung der Erregung notwendige Beweglichkeit der Fibrillensäure aufzuheben.

Auf eine Diskussion dieser Anschauung will ich mich hier nicht einlassen. Es kommt mir zunächst allein darauf an, zu zeigen, dass auch die von Bethe beobachteten Tatsachen zu meiner auf den beschriebenen Versuchen basierenden Theorie passen. Nämlich Bethe's Bild des Elektrotonus wäre folgendermaassen zu erklären: der konstante Strom setzt eine Polarisation an den ionenpermeablen, polarisierbaren Fibrillenoberflächen; die dadurch zustande kommenden, polar verschiedenen, chemischen Veränderungen in der Fibrillen-

1) Allgem. Anat. u. Physiol. d. Nervensystems 1903, Kapitel 14. 
umgebung verursachen an der Anode eine Auflockerung der Fibrillensubstanz, die sich in einer matteren Färbung äussert, vielleicht auch mit wirklicher Verminderung der Fibrillenfärbbarkeit einhergeht, und an der Kathode eine Verdichtung, welche sich entsprechend färberisch äussert. Die Kolloidprozesse der Auflockerung resp. Verdichtung werden durch die Narkotika gehemmt, wie meine Versuche es deutlich gezeigt haben; deshalb verändert sich das Nervenbild nicht durch die Durchströmung mit konstantem Strom.

Auch die meisten übrigen von Bethe beschriebenen Zusammenhänge zwischen Aussehen und Funktion lassen sich auf Grund der Kolloidtheorie erklären.

\section{Zur Theorie der Narkose.}

Die aus den vorstehend beschriebenen Experimenten gezogenen Schlüsse enthalten eine neue Theorie der Narkose, welche besagt, dass narkotisierende Substanzen solche Substanzen sind, welehe die zur Erregung gehörende Zustandsänderung der Protoplasmakolloide zu hemmen vermögen. Nun existiert seit etwa 8 Jahren die auf eine Fülle ausgezeichneter Versuche fundierte Theorie der Narkose von $\mathrm{Hans}$ Meyer und Overton, und es fragt sich, in welchem Verhältnis die beiden Theorien zueinander stehen. Die Theorie von Meyer und Overton bildet im gewissen Sinne einen Spezialfall eines früher gelegentlich von Ehrli $\mathrm{ch}^{1}$ ) ausgesprochenen Satzes, dass Neurotropie und Lipotropie miteinander zusammenhängen; sie besagt, dass Narkotika allgemein solche Stoffe sind, welche in fettähnlichen („lipoiden“) Lösungsmitteln sich. lösen, und dass die narkotische Kraft um so grösser ist, je grösser der Teilungskoeffizient Lipoid: Wasser ist. Da Lipoide aber in allen Zellen enthalten sind, so erklärt die Theorie zugleich, warum die Narkotika nicht bloss "neurotrop“ sind, warum vielmehr alle lebenden Zellen narkotisierbar sind. Diese Lehre, so richtig sie sicherlich ist, befriedigt nun doch nicht, sobald man sich die Frage vorlegt: wie kommt die Zellähmung durch die Narkotika zustande? Was hat die Anbäufung gewisser chemischer Verbindungen in den Zellipoiden mit der Funktionshemmung derselben Zellen zu tun? Es ist gar nicht anders denkbar, als dass bei der Erregung in den Lipoiden etwas vor

1) Constitution, Verteilung und Wirkung chemischer Körper. Leipzig 1893. 
sich geht, was mit den funktionellen Vorgängen im Protoplasma der erregten Substanz zusammenhängt, und dass dieser Prozess, der sich bei der Erregung in den Lipoiden abspielen muss, durch die Narkotika gehemmt werden kann.

Diese Überlegung stellt nun die Vermittlung zwisehen den beiden unabhängig voneinander, von ganz verschiedenen Ausgangspunkten herkommenden Theorien der Narkose dar. Das Hauptlipoid, das in allen Geweben vorkommt, das Lezithin, ist ein Kolloid; von ihm habe ich vorher angegeben, dass sein Verhalten in kolloidaler Lösung gegenüber den Elektrolyten gut mit dem Verhalten der Erregbarkeit und der elektrischen Reaktion von Muskeln gegenüber den Elektrolyten übereinstimmt (siehe Seite 493 ff.). Der notwendig bei der Erregung in den Lipoiden sich abspielende Vorgang ist also offenbar eine kolloidale Zustandsänderung, und der Zusammenhang des Lipoidvorgangs mit den funktionellen Vorgängen im Protoplasma bei der Erregung ist offenbar ein Elektrolytzusammenhang. Nunmehr ist also Narkose folgendermaassen zu definieren: Narkose beruht auf der Ansammlung lipoidlöslicher Stoffe in den Zellipoiden biszu einer bestimmten Konzentration und Aufhebung einer sonst bei der Erregung durch die Zellelektrolyte herbeigeführten Zustandsänderung in den kolloidalen Lipoiden.

Damit erscheint für den Augenblick die Narkose vollständig erklärt, sie erscheint als ein Vorgang, durch den der vielgliedrige Prozess der Erregung an dem einen notwendigen Glied der kolloidalen Zustandsänderung unterbrochen wird.

Man könnte nun nur noch fragen, wie man sich den Einfluss der Narkotika auf das Lezithin denken soll. Offenbar muss doch Tränkung des Lezithins mit Narkotizis sowohl schrumpfende resp. fällende, wie quellende resp. lösende Einflüsse von Elektrolyten zu hemmen vermögen. Dass das erste der Fall ist, ist vorauszusehen, da Lezithin nicht bloss die Narkotika löst, sondern umgekehrt auch Narkotika das Lezithin in Lösung halten; zudem habe ich früher zusammen mit Gord on ${ }^{1}$ ) gezeigt, dass die Ausfällung von Lezithin mit Baryum- oder Calciumsalzen durch Narkotika gehemmt werden kann. Der zweite Fall muss experimentell noch untersucht werden, es ist

1) H ofmeister's Beiträge Bd. 5 S. 432. 1904. 
möglich, dass der Theorie hier noch eine Schwierigkeit entsteht. Auf alle Fälle ist aber schon gezeigt, dass die Kolloide der Achsenzylinder durch Äthylurethan gegen den quellenden Einfluss des Kaliumsulfats unempfindlich werden (s. Seite 509).

\section{Wirkung der Erdalkalisalze auf den Ruhestrom des Muskels.}

Ich schliesse hier noch einige Beobachtungen über die Wirkungen der Erdalkalisalze an, weil sie ja in Beziehung zum Erregungsproblem stehen. Durch kleine Dosen von Kalksalzen kann man bekanntlich die Erregbarkeit herabsetzen; das ist durch die Untersuchungen von Ringer, Locke u. a. für den Muskel, den Nerv und das Herz, durch diejenigen von Mac $\mathrm{Callum}^{\mathbf{1}}$ ) und Martin Fischer ${ }^{2}$ ) für Darm und Nieren festgestellt. Die Kalksalze wirken also äusserlich ähnlich wie Narkotika in schwacher Dosis. Overton ${ }^{3}$ ) hat weiter gezeigt, dass die lähmende Wirkung von Kalium-, Rubidium-, Ammoniumchlorid auf die Muskeln durch kleine Mengen von Calciumchlorid in antagonistischem Sinn beeinflusst werden kann. An diese Beobachtung will ich anknüpfen. Es fragt sich, ob die mit der Salzlähmung einhergehende Negativierung der Muskeln auch mit Hilfe von Calciumsalzen hintangehalten werden kann, oder allgemein: ob überhaupt die stromentwickelnden Fähigkeiten der neutralen Alkalisalze durch Ca gehemmt werden können, so wie sie durch Narkotika gehemmt werden können. Und wenn das der Fall ist, worin besteht dann der Unterschied zwischen einem Narkotikum und dem Kalk in pharmakologischer Beziehung?

Ich habe diese Fragen schon früher ${ }^{4}$ ) experimentell zu behandeln begonnen. Es ergab sich, dass von den Erdalkalien wenigstens $\mathrm{Sr}$ und $\mathrm{Ba}$ die Entwicklung des Kalistroms behindern, während $\mathrm{Ca}$ diese Eigenschaft nicht haben sollte. Ich habe jetzt die Versuche noch einmal aufgenommen und bei genauerer Prüfung gefunden, dass dem $\mathrm{Ca}$ wenigstens eine schwache Hemmungswirkung doch zukommt. Folgende Beispiele mögen das zeigen: siehe Fig. 11, 12, 13 u. 14 .

Entsprechende .Versuche wurden noch mit $\mathrm{KNO}_{3}, \mathrm{RbNO}_{3}$, $\mathrm{NH}_{4} \mathrm{NO}_{3}$ und $\mathrm{NH}_{4} \mathrm{Cl}$ ausgeführt. Die meisten Versuche sind mit $\mathrm{RbCl}$ gemacht.

1) Americ. Journ. of Physiol. 1903 u. 1904.

2) University of California Publications 1903 u. 1904.

3) Pflüger's Arch. Bd. 105 S. 176.1904.

4) Pflüger's Arch. Bd. 106 S. 623.1905. 
Man sieht, dass das Ca eine deutliche schwache Hemmungswirkung ausübt, welche von der Konzentration ziemlich unabhängig zu sein scheint. Der Effekt unterscheidet sich nicht von demjenigen, den die Narkotika ausüben, es sei denn quantitativ, was ich bisher nicht

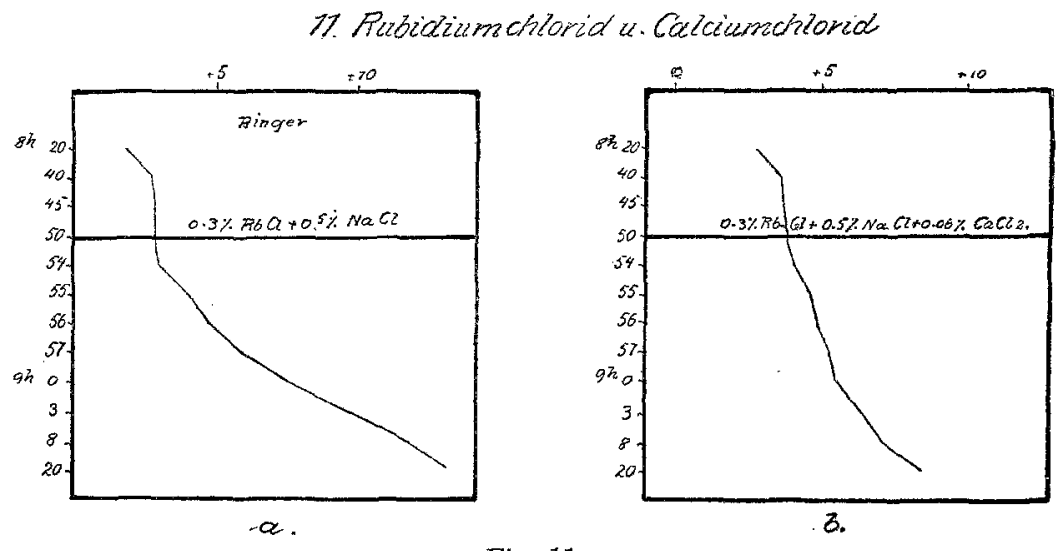

Fig. 11.

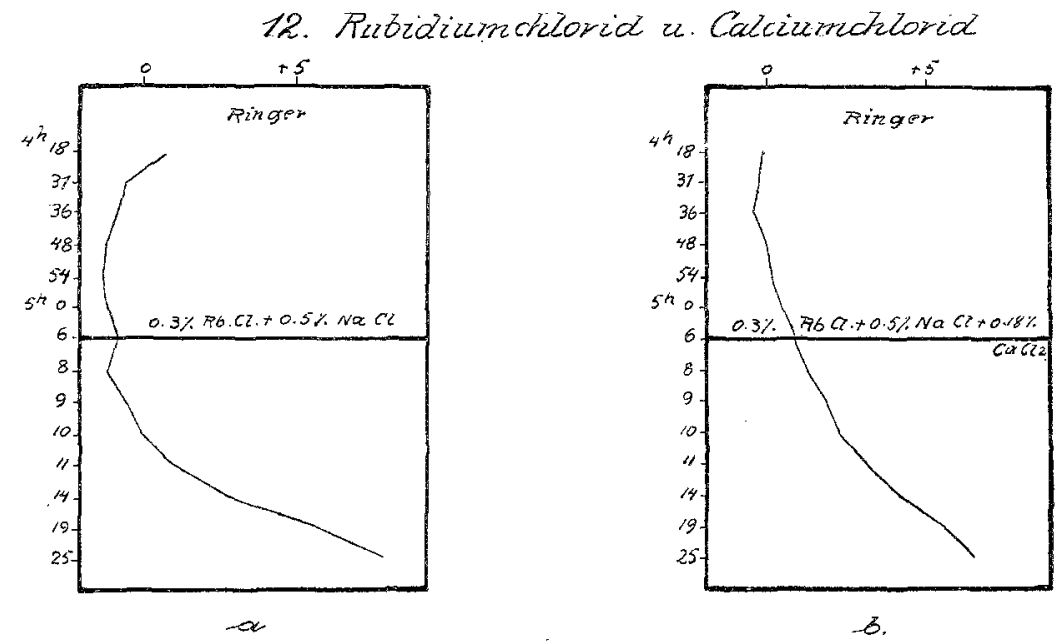

Fig. 12.

mit Bestimmtheit behaupten kann. Also auch nach diesen elektrischen Messungen wirkt das Calcium ähnlich wie ein Narkotikum. Dazu kommt, dass Ca-Salze auf die Protoplasmakolloide, auf Eiweiss und auf Lezithin, einwirken. Es ist also auch annehmbar, dass die Calciumsalze auch an derselben Stelle angreifen wie die Narkotika. Einen deutlichen Untersehied zwischen der Wirkung beider vermag 
Beiträge zur physikalischen Chemie der Erregung and der Narkose. 515 ich überhaupt bis jetzt nicht anzugeben. Versuche, Differenzen in der Reversibilität der beiden Wirkungen nachzuweisen, haben bis jetzt zu keinem deutlichen Ergebnis geführt; ich würde aber doch noch am ersten an Unterschiede der Art denken; es würden sich

13. Fubidiumchlorid und Calciumohiorid
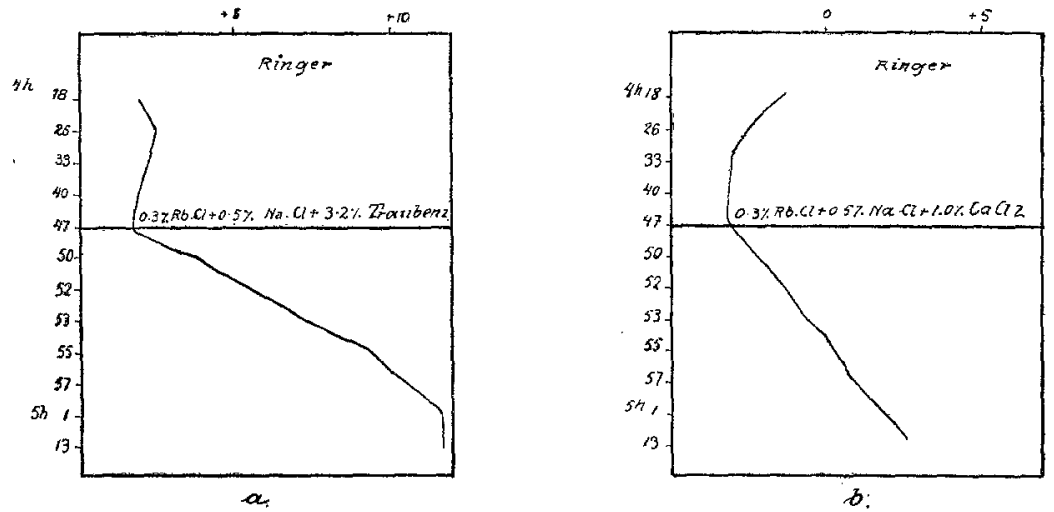

Fig. 13.

14. Natriumjodid und Caloumechlorid.
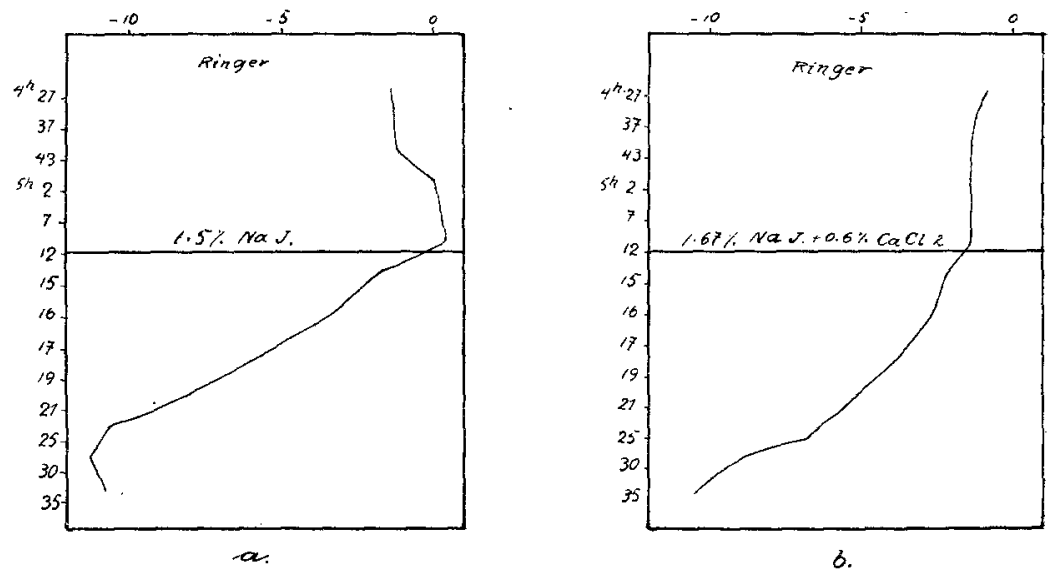

Fig. 14.

dann Ca und die Narkotika ähnlich voneinander unterscheiden, wie etwa die basischen (chemisch aktiven) und die indifferenten (chemisch inaktiven) Narkotika, deren gegenseitige Abgrenzung ja ebenfalls Schwierigkeiten bereitet.

Eine Versuchsreihe mit den den Erdalkalisalzen verwandten Magnesiumsalzen hat keine deutliche Fähigkeit, die Salzruheströme 
$\mathrm{zu}$ beeinträchtigen, ergeben, wie auch entsprechend von $\mathrm{O}$ v e r t o u beobachtet wurde, dass die Mg-Salze keine Antagonisten der K-Salze sind. Diese Untersuchungen waren im Hinblick auf die Angaben von Meltzer ${ }^{1}$ ) über die narkotischen Eigenschaften starker Magnesiumsulfat-Lösungen von Interesse.

\section{Zusammenfassung.}

1. Bei der lokalen Behandlung unverletzter Sartorien mit isotonischen Lösungen neutraler Alkalisalze entstehen Ruheströme von je nach dem Salz verschiedener Spannung und Richtung. Die stromentwickelnden Fähigkeiten der Kationen und Anionen stufen sich dabei, wie neue Versuche zeigen, in der gleichen Richtung ab, in der sich die Fähigkeiten der Ionen, den Lösungszustand von Eiweiss und Lezithin zu beeinflussen, abstufen.

2. Salze, welche den Ruhestrom von regulärer Richtung (LängsQuerschnittstrom) erzeugen, heben die Erregbarkeit auf, Salze, welche keinen oder einen konträren Strom erzeugen, vermögen die Erregbarkeit zu konservieren. Daraus und aus der unter 1. angeführten Tatsache wird der Schluss gezogen, dass Erregung, elektrische Reaktion der Erregung und Kolloidkonsistenz zusammenhängen.

3. Erregung, natürliche elektrische Reaktion bei der Erregung, d. h. Aktionsstrom, und künstliche elektrische Reaktion durch Salze, d. h. Salzruhestrom, werden durch die Narkotika gehemmt. Dem unter 2. ausgesprochenen Schluss entsprechend, wird auch die mit Erregbarkeitsänderung einhergehende Kolloidzustandsänderung (des Achsenzylinders) durch Narkotika gehemmt.

4. Die Narkotika hemmen also den zur normalen Erregung gehörigen Kolloidprozess; im Anschluss an die herrschende Theorie der Narkose von Hans Meyer und Overton ist anzunehmen, dass dieser Kolloidprozess sich im Lezithin abspielt. Narkose beruht also auf Ansammlung lipoidlöslicher Substanz im lipoiden Lezithin bis zu einer bestimmten Konzentration und Sistierung des sich normaler Weise bei der Erregung dort abspielenden Kolloidvorgangs durch diese Substanz.

5. Die Erdalkali-, speziell die Calcium-Salze wirken in mehrfacher Hinsicht ähnlich wie die Narkotika.

1) Amer. Journ. of physiol. vol. 14 p. 366. 1905. Berl. klin. Wochenschr. 1906 S. 73. 